\title{
Algunos problemas sobre la interpretación de valores de contaminación atmosférica: el caso de Madrid
}

\author{
M. ${ }^{a}$ Pilar Borderías Utibeondo
}

\section{INTRODUCCIÓN}

Cuando se acomete un trabajo de investigación desde cualquier ámbito científico, entre otros puntos de partida, son básicos dos aspectos: la recogida de una información veraz, precisa y suficiente, y la existencia de unos baremos que sean aceptados de forma generalizada, de forma que podamos evaluar objetivamente la información obtenida.

En el caso de la investigación del medio atmosférico que hemos ido siguiendo a lo largo de varios años, hemos tropezado con diversos problemas, precisamente en estos dos puntos básicos de partida. Por ello en estos momentos nos planteamos si sólo podremos llegar a un conocimiento meramente aproximativo pero no real de los niveles de contaminación que nos rodean.

Las últimas décadas han supuesto una revolución tecnológica en todos los campos de estudio, desarrollándose paralelamente unas nuevas y cada vez más avanzadas metodologías de adquisición e interpretación de datos. Si bien la depuración de unas técnicas es deseable a nivel científico, también es cierto que al investigador le acarrea una serie de problemas a la hora de llevar a cabo un seguimiento evolutivo de las situaciones que estudia. En el caso de la contaminación atmosférica de Madrid el período de observación es reducido, apenas 25 años, pero a 
lo largo de este período se han modificado las técnicas de análisis, registrándose en cada una de ellas unos valores de contaminación que no son en absoluto comparables. Este hecho nos lleva a una situación de total desconcierto en cuanto a cómo se han modificado los niveles de contaminación a lo largo de estos años, y si realmente han sido satisfactorias las medidas tomadas por la Administración para paliar, que no subsanar, el problema.

Otro hecho que contribuye a complicar la valoración de un análisis de la contaminación atmosférica es la diversidad de enfoques desde los que se estudian los problemas del medio ambiente atmosférico. Estos son observados desde perspectivas ecológicas, biológicas, microbiológicas, físico-químicas o de sanidad, las cuales, en el estado actual del conocimiento científico, discrepan en torno a sus conclusiones y a las medidas oportunas a adoptar.

Si a ello añadimos la gran heterogeneidad de los criterios legislativos en cuanto a los niveles que son aceptables por los individuos (véase anexo 1) el caos es ya total. Este aspecto resulta bastante preocupante puesto que la dispersa y confusa normativa que existe sobre el medio ambiente supone una gran dificultad para el conocimiento del tema; demostrando este hecho, Rodriguez Ramos recogía en Civitas la «legislación sobre el medio ambiente natural». El principal problema de este hecho radica en la dependencia de la función protectora frente a la acción productora y en la dificultad de equilibrio entre el binomio costesbeneficios, que lleva a una falta en la concreción de objetivos, y de standares y niveles comunes en torno a cuáles deben ser las características ambientales.

Las consideraciones que vamos a hacer en este artículo se han planteado a partir del seguimiento de la contaminación atmosférica en Madrid, en donde en pocos años se van a modificar tres veces los métodos de toma de muestras y de análisis, con las consiguientes alteraciones en los valores de contaminación registrados.

\section{MODIFICACIONES EXPERIMENTADAS EN LA RED DE SENSORES DE MADRID}

La fecha de partida en el planteamiento del problema de la contaminación urbana podemos situarla en el año 1963, año en que la Escuela 
Nacional de Sanidad instaló una serie de aparatos de medición en puntos estratégicos de Madrid, con el fin de conocer la media diaria de la contaminación por humos y anhídrido sulfuroso; el primer informe apareció en 1964, y al año siguiente el Ayuntamiento instaló ya su propia red.

Esta primera red de análisis de contaminación disponía de técnicas especiales y altamente precisas, según se manifestaba en el resumen anual de la Delegación de Saneamiento y Medio Ambiente del Ayuntamiento de Madrid. Los aparatos captadores de muestras mensuales estaban situados en terrazas de edificios, escogidos dentro de la ciudad de forma que la red formada por ellos resultase representativa. Por su parte, los aparatos captadores de muestras diarias iban provistos de una bomba aspirante que funcionaba eléctricamente, lo que limitaba la libertad de su ubicación, debiendo localizarse próxima a los semáforos para tomar la corriente de ellos. La toma de muestras se realizaba en ellas a una altura aproximada de dos metros sobre el nivel del suelo; también en el caso de las muestras diarias, según los mencionados resúmenes anuales del Ayuntamiento, se manifestaba que la red de aparatos resultaba representativa. En ambos casos, mensual y diariamente, una flota de vehículos del Servicio, con dotación especializada, recogía las muestras y las llevaba al laboratorio central para su análisis.

Las muestras que se tomaban correspondían: mensualmente, a materia sedimentable e índices de $\mathrm{SO}_{2}$; diariamente, a anhídrido sulfuroso, humos, plomo e hidrocarburos; y con períodos de captación menor se recogían los óxidos de nitrógeno, aldehídos, oxidantes, anhídrido carbónico y monóxido de carbono.

Esta red llegó a contar con un total de 51 estaciones de observación. (Véase anexo 2).

A partir de 1978 comenzó a funcionar la nueva red automática de control, mucho más moderna, con métodos informatizados para el proceso de datos y para la recolección de información, contando además con un sistema telemétrico. El número de estaciones en cambio era menor, comprendiendo sólo 20 estaciones de observación, es decir, 31 menos que en la red anterior. (Véase anexo 3). Igualmente quedó reducido el número de toma de muestras puesto que en ella sólo se recogen $\mathrm{SO}_{2}$, partículas en suspensión y monóxido de carbono, realizándose manualmente las observaciones de plomo. Por otra parte, la ubicación de las mencionadas estaciones ya no era tampoco la misma que en la red anterior, quedando más retiradas de los focos emisores de contaminación. 
Las principales diferencias que llevan a no poder ser relacionados los valores obtenidos por una y otra red en los tres elementos contaminantes que recoge la red actual, se centran en los siguientes aspectos:

- Las observaciones de partículas en la primera red diferenciaban los humos y la materia sedimentable, entendiendo por humos aquellas partículas que por su ínfimo tamaño no tenían velocidad de caída apreciable, por lo que permanecían en la atmósfera durante largos períodos de tiempo. Por materia sedimentable se entendían todas aquellas partículas del aire depositadas por la acción de la gravedad o arrastradas por la lluvia. La toma de estas muestras se realizaba según norma BS $17471 .^{a}$ y $2 .^{a}$ parte respectivamente, con "Depósito Gauge» para las partículas y con filtro Whatman para los humos. Por el contrario, el actual medidor de partículas registra solamente la fracción respirable, es decir, aquellas que cuentan con un diámetro inferior a $10 \mu$, denominadas partículas en suspensión.

Además de estas diferencias en cuanto a su análisis y selección, también hay que contar su distinta ubicación, más próximas a los focos de emisión en el primer caso que en el segundo.

- Las observaciones de monóxido de carbono en la antigua red eran recogidas a través de muestras instantáneas en unas bolsas de plástico especiales rellenadas por medio de un balón Kichardsd, que posteriormente eran analizadas en el laboratorio usando técnicas de infrarrojo no dispersivo. En el caso de la actual red de control, el monóxido de carbono se registra sólo en 5 estaciones a través de medidores, mediante absorción de radiación infrarroja.

- Las obsenvaciones de dióxido de azufre en la anterior red se registraban mensualmente, determinándose su indice según normas BS 1747 parte $4^{\mathrm{a}}$, y su valor era considerado tan sólo como orientativo, con una relativa aproximación. En la actualidad su detección se realiza mediante sensores con métodos basados en la fotometría de llama.

Todas estas modificaciones llevan a los acusados contrastes que veremos y analizaremos en el apartado núm. 2.

En el balance del problema de la contaminación tenemos que, frente a unos focos emisores más o menos constantes, se conjugan una serie de factores atmosféricos variables que modifican la situación ambiental determinando la concentración, duración y frecuencia de la contamina- 
ción. Para el estudio de estos parámetros la nueva red ha supuesto un gran avance, puesto que sus propias estaciones cuentan con aparatos meteorológicos que permiten correlacionar, con mayor precisión, los niveles de contaminación con algunas situaciones meteorológicas de la zona. Concretamente, todas cuentan con un pluviómetro, y en tres de ellas se han emplazado medidores de dirección y velocidad del viento, temperatura y humedad relativa, y en una de ellas se mide también la presión y la insolación.

Pero esta red que acabamos de comentar tampoco va a durar mucho tiempo, puesto que en la actualidad se está abordando nuevamente la renovación de la red de control. Esta renovación parece venir determinada por dos hechos: problemas en el suministro de repuestos para las reparaciones de los actuales aparatos, y la exigencia por parte de la CEE de adaptar los muestreos de la calidad del aire a la normativa comunitaria. En este caso, por lo menos inicialmente, no variará el número de estaciones con respecto a la anterior, tan sólo se incluirá la de la Plaza de Cristo Rey que en la actualidad funcionaba de forma experimental, sin estar incluida en la red.

Los principales aspectos que parece ser que llevará consigo esta nueva red serán:

- Ampliación del número de contaminantes a medir.

- Descentralización, aunque no independencia, del control de la estación central, pudiendo regir su funcionamiento cada una de las estaciones remotas.

- Para el procesamiento de datos se hace referencia a su relación con la «legislación vigente».

- Conexión de la red con la Red Nacional de Vigilancia de la Contaminación Atmosférica.

- En cuanto a las técnicas que se utilizarán se preven:

- Determinación de $\mathrm{SO}_{2}$ mediante fluorescencia de ultravioleta, según exige la Environmental Protección Agency (EPAUSA).

- Determinación de partículas igual que en la red actual, mediante atenuación de radiación $\beta$.

- Determinación de $\mathrm{NO}_{x}$ a través de 11 sensores mediante quimiluminiscencia.

- Determinación de CO en 9 sensores, con igual técnica que la actual (absorción de radiación infrarroja). 
- Determinación de $\mathrm{O}_{3}$ en 4 sensores, mediante absorción de radiación ultravioleta.

- Determinación de hidrocarburos en 2 sensores, mediante ionización de llama.

- Ampliación de los parámetros meteorológicos.

- Determinación de lluvia ácida en 2 monitores.

Vistas estas modificaciones nos planteamos los siguientes interrogantes:

Si en general la orientación de los estudios de contaminación, a través de la interpretación de las muestras recogidas, suelen dirigirse a testificar el grado de deterioro ambiental, y a dar cuenta de la evolución experimentada, ¿cómo podrá ser efectiva una valoración que cuenta con problemas en: "los métodos de análisis" que son condicionantes de la validez de los datos, los cuales se van cambiando con las sucesivas actualizaciones tecnológicas de la red; en "la toma de muestras", que, si bien en cada periodo se han considerado representativas, al instaurar unos nuevos modelos de muestreo quedan desvirtuadas; $y$ en la "standarización de unos valores de referencia" que permita evaluar el grado de toxicidad de los contaminantes?

Si la finalidad de los estudios de contaminación es su posterior utilización en el control del medio ambiente y la orientación de los posteriores planes de saneamiento, ¿cómo podrían ser utilizados estos si las series son cortas y quedan interrumpidas cada decenio?

Evidentemente, de momento, debemos contentarnos con conseguir una valoración meramente aproximativa, especificando en cada caso la metodología utilizada, siguiendo las recomendaciones para estos casos de la American Chemical Society para la mejora del medio ambiente (ACSCEI, 1980), lo cual permite a cada interesado interpretar y establecer su juicio crítico sobre los resultados obtenidos.

En un futuro, puede que la incorporación de España a la CEE permita una mayor rigurosidad de la información registrada, por cuanto que a partir de 1992 deberemos adaptarnos a una normativa general para todos los países comunitarios. En este sentido la CEE cuenta con Directivas y Normativas al respecto, que se orientan a:

- Armonizar las diferencias entre las medidas nacionales, tanto legislativas como administrativas existentes entre los paises miembros, debiendo coordinarse las políticas del medio ambiente entre sí y con los programas comunitarios. 
- Limitación de la contaminación del aire fijando objetivos de calidad y límites en materia de contaminación del aire, haciendo referencia solamente a los valores de inmisión referidos al $\mathrm{SO}_{2}$, particulas en suspensión, plomo, monóxido de carbono, hidrocarburos y óxidos de nitrógeno; también se limita la polución procedente de grandes instalaciones de combustión y de vehículos de motor. Así mismo, la Directiva 80/779 contempla la adopción de valores límites entendidos como concentraciones máximas admisibles durante períodos determinados; esta Directiva establece también las técnicas de muestreo y de análisis a utilizar.

- Fomentar la investigación sobre temas medio-ambientales. En este sentido, en el momento de incorporarse España a la CEE en 1986 se lanzaba el $4 .^{\circ}$ programa de investigación en materia de medio ambiente; como continuación a este programa están a punto de aprobarse dos programas, independientes pero correlacionados, de investigación en el campo del medio ambiente. Estos son los programas STEP (ciencia y tecnología para la protección del medio ambiente, dotado con 10.000 millones de pesetas) y el programa EPOCH (programa europeo de climatología y riesgos naturales, dotado con 5.500 millones de pesetas).

\section{VALORACIÓN DE LOS NIVELES DE CONTAMINACIÓN DE MADRID Y SUS CONTRASTES ENTRE LAS DISTINTAS REDES DE OBSERVACIÓN}

Las valoraciones llevadas a cabo por distintos estudios sobre la contaminación en el área urbana madrileña concluye siempre en la demostración de una situación contaminante evidente, pero no llega a haber una unanimidad en cuanto al grado que alcanza el problema.

Los contaminantes que alcanzan una mayor influencia en el problema atmosférico de Madrid son los humos, el monóxido de carbono, y el anhídrido sulfuroso, aunque hay que mencionar también otros de suma importancia (a pesar de que no son medidos por la red automática de control) como son el plomo, al que la CEE considera como un contaminante de primera categoria, que debe ser observado con prioridad por su efecto tóxico sobre el organismo humano; los óxidos de nitrógeno por su capacidad de provocar sinergismos, contaminante por otro lado en el que 
Madrid se encuentra entre las cuatro provincias españolas de mayor emisión global (según unos trabajos publicados en agosto de 1981 por la asociación de medicina y seguridad en el trabajo de UNESA) y que según los datos que aporta la antigua red, llegaron a alcanzar en algún año elevados niveles, superándose en algunos puntos de la ciudad el límite de situación admisible, como en la Plaza de Colón, Cibeles y Gran Vía; y por último los aldehídos, aunque estos no eran motivo de preocupación en la antigua red.

La evolución que ha experimentado la contaminación desde 1969 hasta 1988 podemos verla en los Cuadros I, II y III, y en los gráficos 1, 2 y 3 . En ellos se aprecian unos valores de contaminación elevados en la etapa 1969-1978, valores que superan con una cierta frecuencia los límites máximos admisibles en la legislación de nuestro país. La evolución de los niveles de contaminación mantenía una línea ascendente en las

CUADRO I. EVOLUCIÓN DE $\mathrm{SO}_{2}\left(\mu \mathrm{g} / \mathrm{m}^{3}\right)$

\begin{tabular}{lrrrrrrrrrrrrrr}
\hline Año & Ene. & Feb. & Mar. & Abr. & May. & Jun. & Jul. & Ago. & Sep. & Oct. & Nov. & Dic. & Media \\
\hline 1970 & 210 & 263 & 215 & 124 & 91 & 65 & 63 & 54 & 59 & 101 & 191 & 359 & 150 \\
1971 & 277 & 246 & 166 & 113 & 86 & 104 & 103 & 72 & 81 & 104 & 249 & 226 & 152 \\
1972 & 215 & 170 & 152 & 95 & 83 & 84 & 87 & 65 & 65 & 102 & 154 & 245 & 126 \\
1973 & 255 & 245 & 185 & 113 & 88 & 89 & 90 & 82 & 99 & 161 & 272 & 280 & 163 \\
1974 & 217 & 188 & 159 & 115 & 101 & 68 & 75 & 71 & 79 & 123 & 233 & 332 & 147 \\
1975 & 249 & 182 & 128 & 91 & 68 & 74 & 105 & 99 & 78 & 125 & 155 & 183 & 129 \\
1976 & 286 & 164 & 141 & 77 & 55 & 79 & 86 & 71 & 67 & 67 & 151 & 113 & 113 \\
1977 & 99 & 82 & 103 & 69 & 56 & 50 & 61 & 65 & 91 & 61 & 132 & 111 & 82 \\
& & & & & & & & & & & & & \\
& & & & & & & & & & & & & \\
1978 & - & 89 & 64 & 28 & 17 & 15 & 15 & 14 & 26 & 40 & 117 & 93 & 49 \\
1979 & 101 & 93 & 81 & 52 & 41 & 37 & 38 & 37 & 44 & 51 & 167 & 145 & 73,9 \\
1980 & 128 & 113 & 70 & 44 & 39 & 36 & 37 & 38 & 42 & 55 & 124 & 179 & 75,4 \\
1981 & 177 & 121 & 72 & 45 & 42 & 43 & 42 & 41 & 44 & 55 & 119 & 86 & 73,9 \\
1982 & 106 & 96 & 71 & 51 & 46 & 49 & 51 & 50 & 46 & 54 & 89 & 119 & 69 \\
1983 & 186 & 113 & 84 & 57 & 55 & 51 & 56 & 51 & 59 & 66 & 82 & 130 & 82,5 \\
1984 & 106 & 99 & 85 & 49 & 48 & 49 & 51 & 49 & 60 & 63 & 87 & 119 & 72,0 \\
1985 & 124 & 95 & 76 & 62 & 56 & 55 & 58 & 61 & 62 & 71 & 96 & 150 & 80,5 \\
1986 & 124 & 100 & 80 & 71 & 63 & 62 & 65 & 67 & 66 & 65 & 110 & 145 & 84,8 \\
1987 & 155 & 107 & 86 & 71 & 62 & 64 & 63 & 67 & 71 & 69 & 106 & 107 & 85,6 \\
1988 & 95 & 91 & 82 & 65 & 58 & 60 & 58 & 54 & 64 & 66 & 95 & 153 & 78,4 \\
\hline
\end{tabular}

FUENTE: Ayto. de Madrid. Delegación de Saneamiento y Medio Ambiente. 
Algunos problemas sobre la interpretación de valores...

CUADRO II. NIVELES DE HUMOS/PARTICULAS ${ }^{\star}\left(\mu \mathrm{q} / \mathrm{m}^{3}\right)$

\begin{tabular}{lrrrrrrrrrrrrrr}
\hline Año & Ene. & Feb. Mar. & Abr. & May. & Jun. & Jul. & Ago. & Sep. & Oct. & Nov. & Dic. & Año \\
\hline 1969 & - & - & - & - & - & - & - & - & - & 140 & 120 & 181 & - \\
1970 & 195 & 226 & 178 & 125 & 137 & 129 & 98 & 137 & 200 & 199 & 261 & 361 & 187 \\
1971 & 235 & 269 & 191 & 184 & 204 & 206 & 228 & 222 & 290 & 325 & 325 & 336 & 251 \\
1972 & 287 & 233 & 240 & 183 & 197 & 192 & 175 & 168 & 254 & 261 & 266 & 290 & 288 \\
1973 & 311 & 283 & 210 & 182 & 193 & 196 & 150 & 182 & 204 & 273 & 347 & 287 & 231 \\
1974 & 275 & 230 & 207 & 189 & 165 & 160 & 155 & 169 & 192 & 233 & 293 & 344 & 218 \\
1975 & 286 & 222 & 189 & 195 & 187 & 193 & 176 & 155 & 220 & 267 & 255 & 261 & 217 \\
1976 & 385 & 242 & 201 & 165 & 175 & 168 & 172 & 151 & 201 & 196 & 249 & 194 & 208 \\
1977 & 131 & 128 & 152 & 128 & 114 & 104 & 99 & 96 & 149 & 143 & 163 & 143 & 129 \\
& & & & & & & & & & & & & \\
1978 & - & 73 & 64 & 47 & 46 & 50 & 50 & 55 & 81 & 74 & 87 & 55 & 62 \\
1979 & 59 & 65 & 60 & 47 & 45 & 58 & 55 & 55 & 55 & 66 & 120 & 94 & 64,9 \\
1980 & 91 & 84 & 60 & 52 & 48 & 36 & 36 & 48 & 48 & 60 & 87 & 99 & 62,4 \\
1981 & 103 & 79 & 57 & 49 & 47 & 40 & 37 & 41 & 48 & 68 & 104 & 58 & 60,9 \\
1982 & 77 & 75 & 61 & 58 & 46 & 47 & 52 & 44 & 48 & 50 & 78 & 84 & 60 \\
1983 & 122 & 72 & 56 & 33 & 44 & 61 & 44 & 40 & 54 & 60 & 55 & 81 & 60,1 \\
1984 & 65 & 66 & 55 & 46 & 41 & 48 & 58 & 49 & 55 & 63 & 51 & 63 & 50,8 \\
1985 & 69 & 64 & 40 & 48 & 42 & 46 & 59 & 54 & 60 & 80 & 67 & 98 & 60,5 \\
1986 & 74 & 53 & 42 & 41 & 45 & 50 & 59 & 47 & 50 & 58 & 79 & 81 & 56,5 \\
1987 & 70 & 59 & 62 & 40 & 48 & 42 & 45 & 57 & 58 & 52 & 55 & 62 & 54,1 \\
1988 & 48 & 50 & 48 & 41 & 39 & 40 & 42 & 41 & 64 & 55 & 56 & 85 & 50,7 \\
\hline
\end{tabular}

FUENTE: Ayto. de Madrid. Delegación de Saneamiento y Medio Ambiente.

* (Hasta 1977 valores considerados como humos; a partir de 1978 valores considerados como particuas).

medias anuales hasta el año 1973, a partir del cual comienza un progresivo descenso hasta 1977, (aunque mensualmente habia oscilaciones entre los años, que hacian variar esta regularidad); como excepciones a esta evolución tenemos el $\mathrm{CO}$ y el $\mathrm{NO}_{x}$ cuyos niveles posteriores a 1973 son iguales o superiores a este año.

A partir de la introducción de la red automática podemos observar un corte total en la evolución de los valores de contaminación, que en todos los casos presentan un salto hacia un descenso. Esto evidentemente hay que atribuirlo fundamentalmente a las nuevas metodologías empleadas en la toma de muestras, aunque también es cierto que se implantaron ciertas medidas reguladoras del tipo de combustible a emplear dentro del casco urbano, que pudieron repercutir también en un descenso. 
M. ${ }^{a}$ PILAR BORDERIAAS URIBEONDO

CUADRO III. EVOLUCIÓN DE LA CONTAMINACIÓN POR CO $\left(\mathrm{mg} / \mathrm{m}^{3}\right)$

\begin{tabular}{lrrrrrrrrrrrrr}
\hline Año & $E$ & $F$ & $M$ & $A$ & $M y$ & $J$ & $J$ & $A g$ & $S$ & $O$ & $N$ & $D$ & $A \bar{N} O$ \\
\hline 1972 & - & - & - & - & - & - & - & - & - & 16 & 15 & 17 & - \\
1973 & 19 & 17 & 16 & 15 & 13 & 15 & 13 & 13 & 13 & 12 & 17 & 23 & 15 \\
1974 & 20 & 20 & 19 & 19 & 15 & 17 & 16 & 16 & 10 & 11 & 12 & 17 & 16 \\
1975 & 17 & 15 & 14 & 14 & 15 & - & - & - & 16 & 20 & 23 & 22 & 17 \\
1976 & - & - & - & - & - & - & - & - & - & - & - & - & - \\
1977 & 16 & 13 & 13 & 12 & 11 & 14 & 18 & 14 & 17 & 21 & 21 & 19 & 16 \\
& & & & & & & & & & & & & \\
1978 & - & 0,9 & 0,4 & 0,3 & 1,6 & 2,7 & 3,5 & 4,8 & 5,7 & 0,1 & 0,6 & 0,5 & 1,9 \\
1979 & 0,5 & 0,4 & 0,5 & 0,3 & 1,0 & 3,3 & 2,5 & 0,9 & 3,8 & 4,7 & 6,6 & 6,8 & 2,6 \\
1980 & 5,0 & 4,2 & 2,8 & 2,1 & 3,6 & 3,6 & 3,0 & 3,0 & 4,0 & 6,3 & 6,6 & 6,6 & 4,2 \\
1981 & 7,7 & 5,6 & 5,4 & 3,6 & 3,5 & 4,0 & 3,3 & 2,9 & 5,1 & 5,9 & 8,4 & 5,8 & 5,1 \\
1982 & 5,7 & 5,8 & 4,5 & 3,9 & 4,4 & 4,9 & 4,6 & 3,6 & 4,5 & 5,2 & 5,5 & 5,5 & 4,8 \\
1983 & 8,2 & 5,4 & 6,3 & 5,8 & 5,0 & 5,3 & 4,6 & 4,1 & 7,2 & 8,0 & 7,5 & 7,6 & 6,2 \\
1984 & 6,4 & 6,2 & 5,6 & 3,7 & 4,1 & 4,7 & 4,4 & 3,7 & 5,8 & 6,7 & 5,6 & 6,3 & 5,2 \\
1985 & 7,1 & 6,5 & 5,5 & 6,1 & 5,2 & 5,4 & 4,8 & 4,6 & 5,9 & 8,5 & 7,2 & 9,5 & 6,3 \\
1986 & 8,1 & 7,5 & 6,5 & 6,5 & 6,8 & 7,2 & 7,2 & 5,1 & 6,2 & 6,9 & 7,8 & 7,3 & 6,9 \\
1987 & 7,7 & 6,7 & 5,8 & 6,1 & 5,6 & 6,1 & 5,2 & 5,5 & 7,0 & 6,4 & 6,9 & 8,3 & 6,4 \\
1988 & 7,1 & 6,2 & 5,2 & 5,7 & 6,0 & 5,5 & 6,2 & 5,0 & 6,1 & 7,0 & 5,3 & 5,9 & 5,9 \\
\hline
\end{tabular}

FUENTE: Ayto. de Madrid. Delegación de Saneamiento y Medio Ambiente.

En el período 1978-88, analizado ya por la red automática, se produce una diferenciación en la evolución media anual de los tres contaminantes medidos automáticamente: las oscilaciones interanuales son más moderadas; así los niveles de partículas en suspensión muestran una pequeña oscilación, con una máxima oscilación de $14,2 \mu \mathrm{g} / \mathrm{m}^{3}$ frente a la oscilación de $159 \mu \mathrm{g} / \mathrm{m}^{3}$ de la red anterior, con una tendencia a disminuir en los últimos años; el $\mathrm{SO}_{2}$, por el contrario, ha experimentado un incremento en los últimos años y su oscilación en el período abordado es superior que en el de las partículas, de $36,6 \mu \mathrm{g} / \mathrm{m}^{3}$, aunque inferior a los de la antigua red $\left(81 \mathrm{\mu g} / \mathrm{m}^{3}\right)$; finalmente el CO experimenta también un incremento desde $1,9 \mathrm{mg} / \mathrm{m}^{3}$ hasta alcanzar los $6,9 \mathrm{mg} / \mathrm{m}^{3}$

En cuanto a la evolución anual, si bien los valores absolutos difieren notablemente, su desarrollo a lo largo de los meses es semejante; así se registran los máximos niveles de contaminación en los meses invernales debido a las condiciones atmosféricas de la época y al incremento de los focos emisores de dicha estación. La permanencia anual de las situaciunes contaminantes varían notablemente de unos años a otros, en estre- 
HUMOS/PARTICULAS EN SUSPENSIÓN

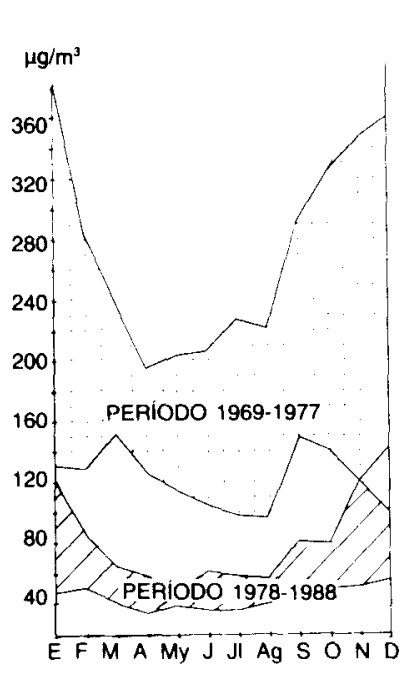

Grático 1

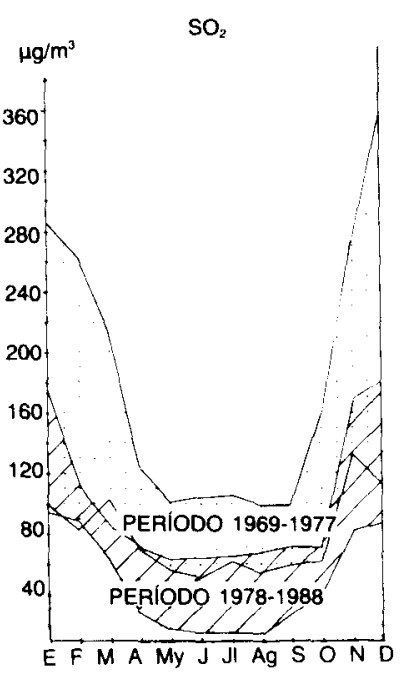

Gráfico 2

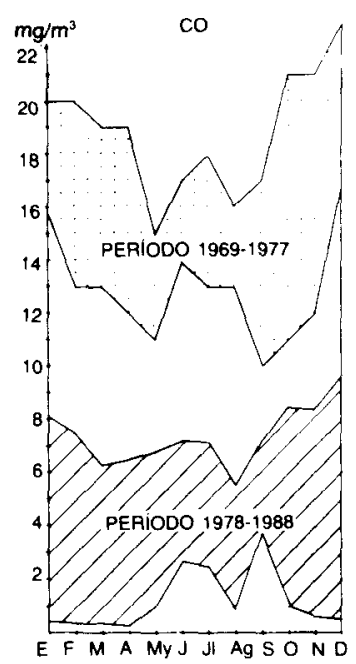

Gráfico 3

EVOLUCIÓN ANUAL DE LA CONTAMINACIÓN EN MADRID: valores máximos y mínimos registrados en la $1{ }^{a}$ Red de observación (periodo 1969-77) y en la red automática de control (periodo 1978-88) para las particulas en suspensión y humos (gráfico $n{ }^{\circ} 1$ ), el $\mathrm{SO}_{2}$ (gráfico $n .^{\circ}$ 2) y el CO (gráfico $n .^{\circ} 3$ ). Obsérvese los marcados contrastes que presentan los valores de las 2 redes de observación.

cha relación con las condiciones meteorológicas. Con datos de la antigua red, según un año muestra, la contaminación por humos, que supera el límite admisible, puede abarcar a todos los meses del año excepto a abril; el monóxido de carbono ( $\mathrm{CO}$ ) entre los meses de octubre y abril, y el anhídrido sulfuroso $\left(\mathrm{SO}_{2}\right)$ entre octubre y marzo. La situación suele agudizarse especialmente de noviembre a enero, y asi, por ejemplo, la situación fue especialmente grave en enero de 1975 y de 1976 y en noviembre y diciembre de 1979 que obligó a declarar "situación de emergencia» a las autoridades municipales.

En el caso de la red automática, la contaminación provocada por los tres contaminantes registrados, $\mathrm{SO}_{2}$, partículas en suspensión y $\mathrm{CO}$, según la legislación vigente en nuestro país, sólo supera el límite admisible en la tabla de valores máximos alcanzados en un dia (véase Cuadro IV) cuyos valores se sobrepasan en alguna ocasión en todos los meses del año, en cambio no se aprecia esa situación de peligro en las medias 
CUADRO IV. VALORES MÁXIMOS ALCANZADOS

\begin{tabular}{|l|r|r|r|r|r|r|r|r|r|r|r|r|}
\hline & \multicolumn{1}{|c|}{$E$} & $F$ & $M$ & $A$ & $M y$ & $J$ & $J$ & $A g$ & $S$ & $O$ & $N$ & \multicolumn{1}{c|}{$D$} \\
\hline $\mathrm{SO}_{2}$ & & & & & & & & & & & & \\
1984 & 1.931 & 1.022 & 841 & 431 & 395 & 247 & 310 & 276 & 491 & 668 & 1.311 & 1.278 \\
1985 & 1.305 & 803 & 1.139 & 445 & 686 & 289 & 559 & 547 & 435 & 582 & 829 & 2.506 \\
1986 & 1.413 & 896 & 614 & 833 & 755 & 254 & 323 & 382 & 326 & 291 & 872 & 1.137 \\
1987 & 1.507 & 889 & 699 & 653 & 232 & 347 & 667 & 612 & 555 & 733 & 1.138 & 869 \\
1988 & 1.241 & 1.018 & 696 & 1.897 & 286 & 685 & 665 & 265 & 646 & 519 & 1.062 & 1.524 \\
\hline Parti- & & & & & & & & & & & & \\
culas & & & & & & & & & & & & \\
1984 & 799 & 1.038 & 695 & 487 & 644 & 727 & 981 & 440 & 450 & 517 & 686 & 638 \\
1985 & 920 & 802 & 522 & 655 & 729 & 816 & 488 & 544 & 937 & 1.308 & 584 & 1.771 \\
1986 & 897 & 1.106 & 436 & 598 & 804 & 627 & 502 & 570 & 735 & 840 & 997 & 1.393 \\
1987 & 1.808 & 1.345 & 674 & 701 & 890 & 778 & 460 & 466 & 655 & 527 & 520 & 694 \\
1988 & 810 & 752 & 924 & 539 & 564 & 469 & 393 & 483 & 974 & 549 & 883 & 1.112 \\
\hline $\mathrm{CO}$ & & & & & & & & & & & & \\
1984 & 39,8 & 44,1 & 74,8 & 30,5 & 21,9 & 36,4 & 44,6 & 62,2 & 80,4 & 74,5 & 60,0 & 52,4 \\
1985 & 47,1 & 77,6 & 36,7 & 40,3 & 78,2 & 33,7 & 55,5 & 50,8 & 75,9 & 92,8 & 78,0 & 88,3 \\
1986 & 43,7 & 37,9 & 35,4 & 26,3 & 52,7 & 34,7 & 44,7 & 32,2 & 47,2 & 45,1 & 53,6 & 50,2 \\
1987 & 51,4 & 34,6 & 38,7 & 31,7 & 24,8 & 44,4 & 25,5 & 45,0 & 61,2 & 40,4 & 52,3 & 38,9 \\
1988 & 45,3 & 36,8 & 34,2 & 26,0 & 26,5 & 30,8 & 31,5 & 26,9 & 52,0 & 45,0 & 35,0 & 42,8 \\
\hline
\end{tabular}

Fuente: Ayuntamiento de Madrid. Delegación de Saneamiento y Medio Ambiente.

mensuales del conjunto de la red, tan sólo ocasionalmente se rebasa en alguna de las estaciones de observación como Quevedo, Plaza de Manuel Becerra, Plaza de Luca de Tena y Paseo de Recoletos. Con respecto a la media anual ocurre lo mismo, mientras que según la antigua red se sobrepasaban los niveles admisibles de contaminación anual en los tres contaminantes (humos, $\mathrm{CO}$ y $\mathrm{SO}_{2}$ ), esto no ocurre en ningún caso en la red automática.

Dentro de la valoración que se hace de los niveles de contaminación, resultan de interés los estudios realizados en torno a la correlación que existe entre unos elevados niveles y el efecto que se produce en determinados grupos de población (Bustawa y Rjazanov, 1962; Frank, 1962; Bates y Hazucha, 1973; Schimmel y Greenberg, 1972; OMS, 1972; Martin; Lawther; Reid; Zikova; Goldsmith y Hexter...). Estos nos ofrecen una valoración aproximativa de la adaptación del individuo a las diferentes condiciones ambientales. Efectivamente las modificaciones ambientales provocadas por la actividad urbana en los últimos decenios ha llevado consigo problemas de adaptación, variable según las respuestas indivi- 
duales, que se manifiestan en una gran diversidad de impactos, agresiones y cambios (véase Anexo 4).

En general parece ser que los gases vertidos al aire no provocan el comienzo de una enfermedad, pero sí pueden provocar una agudización de ciertos trastornos en personas con enfermedades cardio-respiratorias o con tendencias cancerígenas; al tiempo que se producen determinadas molestias en gran número de personas. En función de estos baremos y teniendo en cuenta los valores alcanzados en nuestra ciudad, hemos estimado la incidencia que puede tener sobre la población. Asimismo hemos observado en los "episodios contaminantes" la posible incidencia sobre el aumento de la morbilidad o de la mortalidad.

Adaptándonos al método Brasser, según el cual se establece una relación entre la dosis, el tiempo de exposición, y el efecto del contaminante para el $\mathrm{SO}_{2}$, se observa que los niveles que suelen alcanzarse entre los meses de noviembre a febrero pueden tener una incidencia sobre enfermos con afecciones cardio-respiratorias, e incluso en los valores que quedan registrados por la anterior red de medición, en algunos años, se da algún mes en que los valores alcanzados por el $\mathrm{SO}_{2}$ pudieron aumentar los índices de mortalidad en este tipo de enfermos.

La valoración establecida sobre épocas de episodios de elevada contaminación ha dado las siguientes muestras:

En el episodio de enero de 1975, episodio en el que el Servicio de Información del Instituto Nacional de Toxicología afirmó que la contaminación producida en estos días suponía un «riesgo grave para la salud", hemos determinado, según los baremos aceptados en Inglaterra en que se considera que las concentraciones que superan los $250 \mu \mathrm{g} / \mathrm{m}^{3}$ en humos, y los $500 \mu \mathrm{g} / \mathrm{m}^{3}$ en $\mathrm{SO}_{2}$ para un período de 24 horas producen empeoramientos en enfermos crónicos de pulmón, que en un total de cuatro días se produjo esta situación.

En el episodio de noviembre-diciembre de 1979, estudiado por Sánchez Murias, se comparó la diferencia entre los ingresos y las asistencias con diagnóstico de enfermedades respiratorias durante el episodio y en los días anteriores y posteriores apreciándose unas significativas diferencias aunque no se observó un incremento de la mortalidad.

A estos episodios cabría añadir el problema de las zonas de peligrosidad continuada. Así, por ejemplo, en un estudio epidemiológico de niveles de plomo, realizados por los servicios de medicina interna e higiene industrial del Instituto Territorial de Madrid sobre una zona industrial de la capital, se llegó a la conclusión de que existían unas diferencias significativas en los niveles de $\mathrm{Pb}$ en sangre, entre el medio urbano y el 
rural, con las siguientes diferencias: la tasa media de $\mathrm{Pb}$ en sangre en la zona urbana industrial fue de $18,2 \mu \mathrm{g} / 100 \mathrm{ml}$, mientras que en la zona rural fue de $14,6 \mu \mathrm{g} / 100 \mathrm{ml}$.

Otros métodos de reafirmar una situación contaminante preocupante es el estudio de los efectos sobre las plantas, que tienen un importante papel depurador ambiental; éstas a su vez se ven afectadas por los efectos tóxicos de la contaminación que se evidencia, según estudios hechos por Verdejo Vivas, G., por reducción del crecimiento, disminución del tamaño foliar, variación de las dimensiones de los tallos, alteración de la floración, senescencia prematura, y anillos de los leños más estrechos. En la selección de las especies ornamentales de la ciudad se tiene en cuenta esta incidencia y así para las especies arboreas de las calles suelen escogerse las de hoja caduca para así renovar anualmente sus hojas deterioradas por la contaminación, y con preferencia de hojas pequeñas. En cuanto a las flores tiene que hacerse una gran selección puesto que algunas plantas no pueden florecer en estos espacios como se ha comprobado en Madrid con los pensamientos y las petunias.

Los efectos de la contaminación sobre los edificios se observa a simple vista: los construidos de roca calcárea sufren el denominado "cáncer de la piedra" que ocasiona grandes costos para su restauración, otros edificios ennegrecen, y la casi totalidad de los conjuntos artísticos y monumentales deben sufrir repetidas limpiezas, hecho que a su vez implica un deterioro adicional.

\section{CONCLUSIONES}

Los puntos que hasta aquí han sido mencionados, nos muestran que la línea de investigación cuantitativa de la contaminación atmosférica de nuestra ciudad debe ser acometida con cautela, debiendo aceptarse que hasta el momento sólo puede representar un mero tanteo que no llega a determinar una realidad precisa. Es evidente que la tecnología empleada hasta el momento y los criterios administrativos sobre la calidad del medio ambiente atmosférico establecidos en nuestro país, no permiten considerar esta información como absolutamente rigurosa. No obstante el futuro resulta más prometedor al incorporarse nuestro Servicio de Control Ambiental a tecnologías y baremos homologados con el resto de los países comunitarios. 
En cuanto a la línea de investigación cualitativa, que ha tratado de definir la calidad del entorno atmosférico en relación con las agresiones que se apreciaban en la ciudad y en los seres que en ella viven, podemos decir que ha permitido mostrar la evidencia de un grave problema contaminante y la necesidad de poner urgentes medidas de saneamiento, medidas que han sido dictaminadas por nuestro Ayuntamiento con mayor o menor efectividad según los casos. 
ANEXO 1. LÍMITES DE CALIDAD DEL AIRE EN DIVERSOS PAÍSES

\begin{tabular}{|c|c|c|c|}
\hline \multirow[b]{2}{*}{ Paises } & \multicolumn{3}{|c|}{ Contaminantes } \\
\hline & $\begin{array}{c}\mathrm{SO}_{2} \mu \mathrm{\mu g} / \mathrm{m}^{3} \\
\text { media anual }\end{array}$ & $\begin{array}{l}\text { Particulas } \\
\mu g / m^{3} \\
\text { media anual }\end{array}$ & $\begin{array}{c}\mathrm{CO} \mathrm{mg} / \mathrm{m}^{3} \\
\text { periodo de } 8 \\
\text { horas }\end{array}$ \\
\hline Bélgica & 150 & - & - \\
\hline España $\ldots \ldots \ldots \ldots \ldots$ & 150 & 130 & 15,0 \\
\hline Canadá (nivel aceptable) .... & 60 & 70 & 15,0 \\
\hline Canadá (nivel deseable) $\ldots . .$. & 30 & 60 & \\
\hline Finlandia $\ldots \ldots \ldots \ldots \ldots \ldots$ & 180 & - & 10,0 \\
\hline Alemania ........... & - & - & 10,0 \\
\hline USA ....... & 80 & 75 & 10,0 \\
\hline Israel ...... & - & 75 & 11,5 \\
\hline Argentina & - & - & 11,5 \\
\hline Japón ....... & - & - & 23,0 \\
\hline Italia $\ldots \ldots \ldots \ldots \ldots$ & - & - & 23,0 \\
\hline OMS $\ldots \ldots \ldots \ldots \ldots$ & 60 & 40 & 10,0 \\
\hline OCDE . & 80 & 80 & — \\
\hline EPA ... & - & - & 9 ppm \\
\hline
\end{tabular}

LÍMITE DE CALIDAD DEL AIRE

(Valores 24 horas) $\left(\mu \mathrm{g} / \mathrm{m}^{3}\right)$

\begin{tabular}{lccc}
\hline & $\mathrm{SO}_{2}$ & Particulas & $\mathrm{CO}$ \\
\hline OCDE $\ldots \ldots \ldots \ldots \ldots \ldots \ldots \ldots$ & 250 & 250 & - \\
España $\ldots \ldots \ldots \ldots \ldots \ldots \ldots \ldots$ & 400 & 300 & $45(30$ minutos $)$ \\
OMS $\ldots \ldots \ldots \ldots \ldots \ldots \ldots \ldots$ & - & - & 40 (mg/1 hora) \\
Inglaterra $\ldots \ldots \ldots \ldots \ldots \ldots \ldots$ & 500 & 250 & - \\
EEUU $\ldots \ldots \ldots \ldots \ldots \ldots \ldots \ldots$ & 365 & 260 & $\overline{-}$ \\
URSS $\ldots \ldots \ldots \ldots \ldots \ldots \ldots \ldots$ & $0,05 \mathrm{ppm}$ & $0,5 \mathrm{ppm}$ & $0,8 \mathrm{ppm}(24$ horas $)$ \\
\hline
\end{tabular}

Fuente: Varios autores.

LIMITES MÁXIMOS ADMISIBLES SEGÚN DECRETO DE PROTECCION DEL AMBIENTE ATMOSFÉRICO EN NUESTRO PAÍS

\begin{tabular}{lrrcc}
\hline & $\mathrm{SO}_{2}$ & Partículas & $\mathrm{CO}$ \\
\hline Promedio máximo 2 horas & $\ldots . .$. & 438 & - & $15 \mathrm{mg} / \mathrm{m}^{3} / 8 \mathrm{~h}$ \\
Concentración media diaria $\ldots \ldots$. & 250 & 150 & $45 \mathrm{mg} / \mathrm{m}^{3} / 30$ minutos \\
Concentración media mensual $\ldots$ & 151 & 100 & - \\
Concentración media anual $\ldots . .$. & 80 & 80 & - \\
\hline
\end{tabular}


Algunos problemas sobre la interpretación de valores...

\section{ANEXO 2}

EMPLAZAMIENTO DE LAS ESTACIONES DE OBSERVACIÓN MENSUAL (hasta 1977)

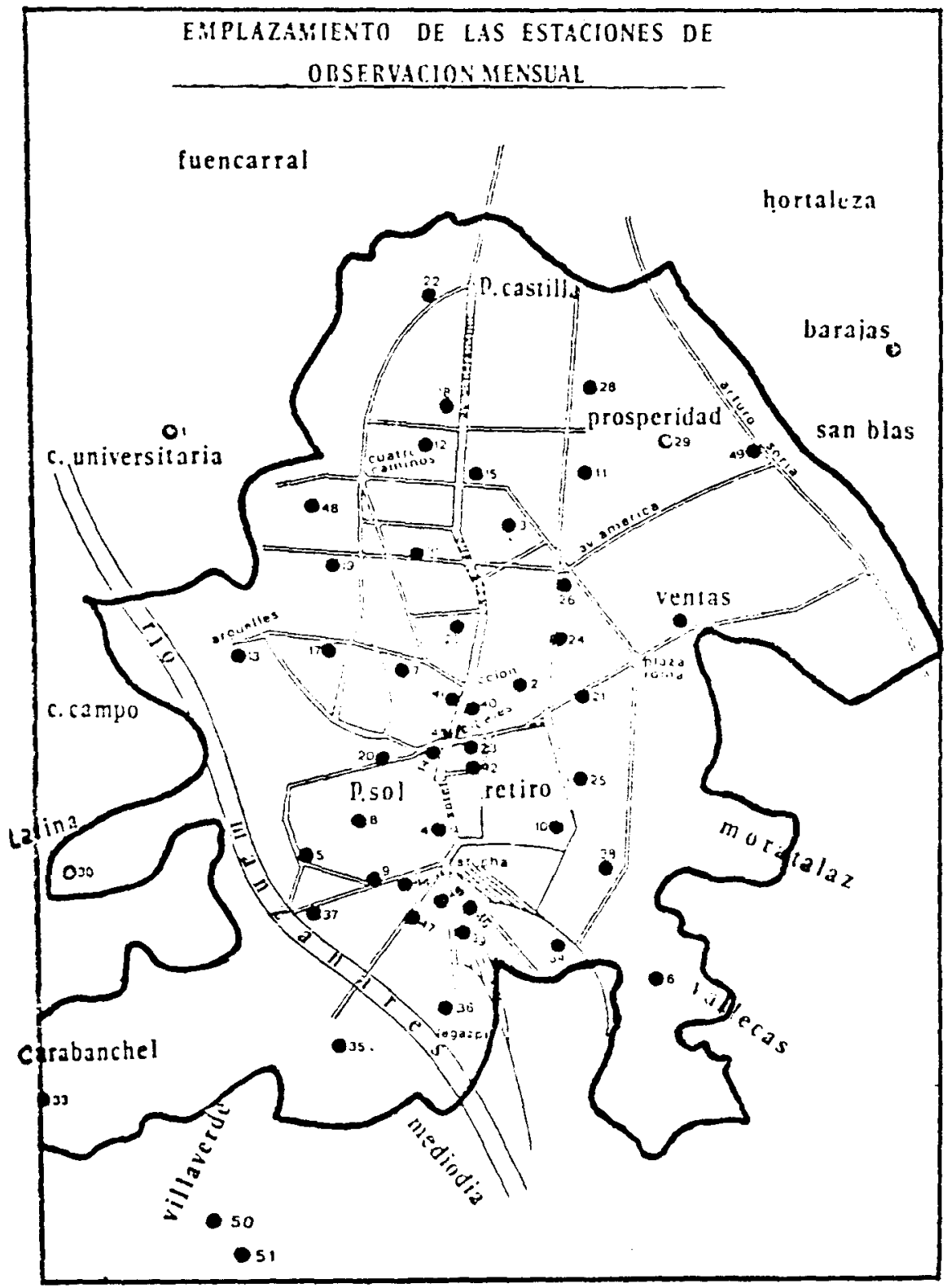


ANEXO 2.1

EMPLAZAMIENTO DE LAS ESTACIONES DE OBSERVACIÓN DIARIA (hasta 1977)

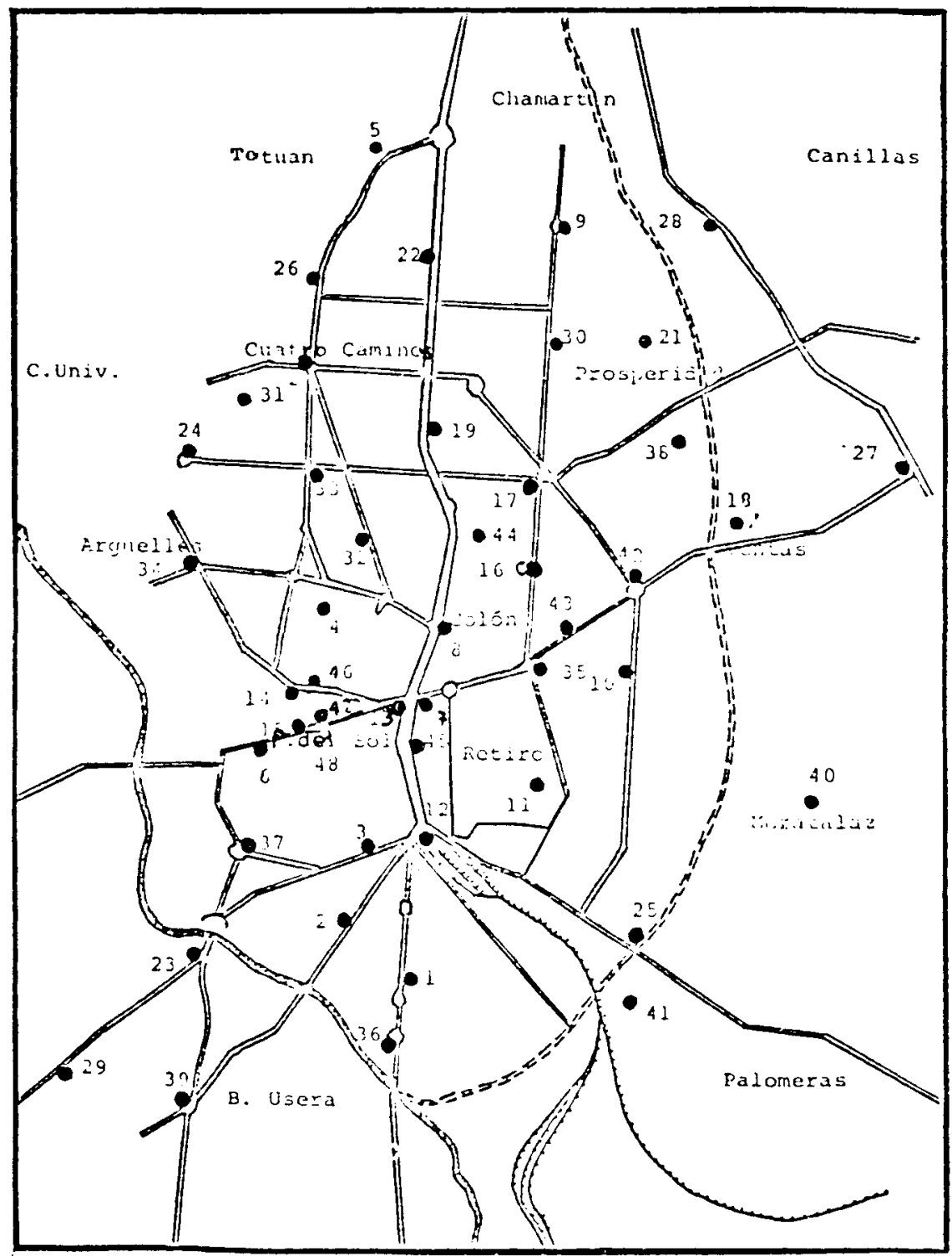




\section{ANEXO 2.2}

\section{EMPLAZAMIENTO DE LAS ESTACIONES DE OBSERVACION MENSUAL (hasta 1977)}

N. ${ }^{\circ}$ 1. Facultad de Farmacia. Ciudad Universitaria.

N. ${ }^{\circ}$ 2. Tenencia de Alcaldía de Buenavista. Velázquez, 52.

N. ${ }^{\circ}$ 3. Instituto Nacional de Quimica Orgánica. Juan de la Cierva, 1.

N. ${ }^{\circ}$ 4. Tenencia de Alcaldía de Retiro. Paseo del Prado, 30.

N. ${ }^{\circ}$ 5. Parque de bomberos. Puerta de Toledo.

N.. 6. Equipo quirúrgico. Concordia, 15 (Vallecas).

N. ${ }^{\circ}$ 7. Instituto Municipal de Educación. Mejía Lequeríca, 21.

N. ${ }^{\circ}$ 8. Imprenta Municipal. Concepción Jerónima, 9.

N. ${ }^{\circ}$ 9. Casa de Baños. Glorieta de Embajadores.

N. ${ }^{\circ}$ 10. Observatorio Central Meteorológico. Parque del Retiro.

N. ${ }^{\circ}$ 11. Academia Nacional José Antonio. Mantuano, 52.

N. ${ }^{\circ}$ 12. General Moscardó, 21.

N..$^{\circ}$ 13. Instituto Nacional de Estadística. Ferraz, 41.

N. ${ }^{\circ}$ 14. Jefatura de FET. Alcalá, 44.

N. ${ }^{\circ}$ 15. Instituto Nacional de Colonización. Avda. del Generalísimo, 2.

N. ${ }^{\circ}$ 16. Mutualidad Nacional de Administración Local. Londres, 60.

N. ${ }^{\circ}$ 17. ICAl. Alberto Aguilera, 23.

N. ${ }^{\circ}$ 18. Ministerio de Información y Turismo. Avda. del Generalísimo, 39.

N. ${ }^{\circ}$ 19. Parque Móvil de Ministerios. Céa Bermúdez, 5.

N. ${ }^{\circ}$ 20. Ministerio de Hacienda. Alcalá, 3.

N..$^{\circ}$ 21. Faros y Balizas. Alcalá, 94.

N..$^{\circ}$ 22. Tenencia de Alcaldía de Tetuán. Bravo Murillo, 257.

N. ${ }^{\circ}$ 23. Instituto Nacional de Previsión. Alcalá, 56.

N. ${ }^{\circ}$ 24. Instituto Nacional de Industria. PI. Marqués de Salamanca.

N. ${ }^{\circ}$ 25. Hospital del Niño Jesús. Menéndez Pelayo, 65.

N. ${ }^{\circ}$ 26. Instituto de Pedagogia Terapéutica. General Oráa, 49.

N. ${ }^{\circ}$ 27. Comisaría General de Abastecimientos y Transportes. Almagro, 21.

N. ${ }^{\circ}$ 28. Chile, 6 .

N. ${ }^{\circ}$ 29. Morris. López de Hoyos, 171.

N. ${ }^{\circ}$ 30. Cuartel Guardia Civil. P. ${ }^{\circ}$ Extremadura, 115-117.

N. ${ }^{\circ}$ 31. Senvicio Social. General Sanjurgo, 39.

N. ${ }^{\circ}$ 32. Aeropuerto de Barajas.

N. ${ }^{\circ}$ 33. Residencia Carmen Polo de Franco. Carabanchel Bajo.

$\mathrm{N} .{ }^{\circ}$ 34. Huarte. Explosión, $\mathrm{s} / \mathrm{n}$.

N. ${ }^{\circ}$ 35. Escuelas Luis Moscardó. General Marvá.

N. ${ }^{\circ}$ 36. Matadero Municipal. Legazpi.

N. ${ }^{\circ}$ 37. Paseo de las Acacias, 65. Gta. de las Pirámides.

N. ${ }^{\circ}$ 38. Doctor Esquerdo, 138. 
N. ${ }^{\circ}$ 39. Tabacalera. Bustamante, General Lacy.

N. ${ }^{\circ}$ 40. Paseo Calvo Sotelo, 20.

N. ${ }^{\circ}$ 41. Paseo Calvo Sotelo, 17.

N. ${ }^{\circ}$ 42. Alfonso XII, 20.

N. ${ }^{\circ}$ 43. Paseo Calvo Sotelo, 4.

N. ${ }^{\circ}$ 44. Escuela de Peritos Industriales. Paseo General Primo de Rivera.

N. ${ }^{\circ}$ 45. Banco de Vizcaya, c/ Vizcaya.

N. ${ }^{\circ}$ 46. Electrolux. Méndez Álvaro, s/n.

N. ${ }^{\circ}$ 47. Galerías Preciados. Paseo Santa María de la Cabeza.

N. ${ }^{\circ}$ 48. Auxilio Social. Avda. del Valle.

N. ${ }^{\circ}$ 49. Auxilio Social. Arturo Soria.

N. ${ }^{\circ}$ 50. Standard Eléctrica. Villaverde.

N. ${ }^{\circ}$ 51. Hotel Olimpo. Carretera de Andalucia. 


\section{ANEXO 2.3. \\ EMPLAZAMIENTO DE LAS ESTACIONES DE OBSERVACION DIARIA (hasta 1977)}

N. ${ }^{\circ}$ 1. C/ Ferrocarril, esquina a Paseo Delicias.

N. ${ }^{\circ}$ 2. Palos de Moguer, esquina a Pseo Santa Maria de la Cabeza.

N. ${ }^{\circ}$ 3. General Primo de Rivera, esquina a C/ Valencia.

N. ${ }^{\circ}$ 4. Delegación Divino Pastor, 9.

N. ${ }^{\circ}$ 5. Tenencia de Alcaldía de Tetuán. Bravo Murillo.

N. ${ }^{\circ}$ 6. Plaza de la Villa.

N. ${ }^{\circ}$ 7. C/ Alcalá, esquina a Alfonso XIl.

N. ${ }^{\circ}$ 8. Plaza de Colón.

N. ${ }^{\circ}$ 9. Plaza República Dominicana.

N. ${ }^{\circ}$ 10. Doctor Esquerdo, esquina a O'Donnell.

N. ${ }^{\circ} 11$. Retiro.

N. ${ }^{\circ}$ 12. Glorieta de Carlos V.

N. ${ }^{\circ}$ 13. Cibeles.

N. ${ }^{\circ}$ 14. Callao, esquina a José Antonio.

N. ${ }^{\circ}$ 15. Arenal, esquina a Puerta del Sol.

N. ${ }^{\circ}$ 16. Plaza Marqués de Salamanca.

N. ${ }^{\circ}$ 17. María de Molina, esquina a Gral. Pardiñas.

N. ${ }^{\circ}$ 18. Plaza de José Banús.

N. ${ }^{\circ}$ 19. Vitrubio, esquina a San Juan de la Cruz.

N. ${ }^{\circ}$ 20. Glorieta de Cuatro Caminos.

N. ${ }^{\circ}$ 21. López de Hoyos, esquina a Alfonso XIII.

N. ${ }^{\circ} 22$. Plaza de Cuzco.

N. ${ }^{\circ}$ 23. Plaza Marqués de Vadillo.

N. ${ }^{\circ}$ 24. Plaza de Cristo Rey.

N. ${ }^{\circ}$ 25. Tenencia de Alcaldía de Vallecas. Avda. de la Albufera.

N. ${ }^{\circ}$ 26. Bravo Murillo, esquina a Francos Rodriguez.

N. ${ }^{\circ}$ 27. García Noblejas, esquina a Alcalá.

N. ${ }^{\circ}$ 28. López de Hoyos, esquina a Arturo Soria.

N. ${ }^{\circ}$ 29. General Ricardos, esquina a Oporto.

N. ${ }^{\circ}$ 30. Plaza de Cataluña.

N. ${ }^{\circ}$ 31. Avda. del Valle.

N. ${ }^{\circ}$ 32. Plaza de Chamberí.

N. ${ }^{\circ}$ 33. Cea Bermúdez, esquina a Bravo Murillo.

N. ${ }^{\circ}$ 34. Marqués de Urquijo, esquina a Princesa.

N. ${ }^{\circ}$ 35. O'Donnell, esquina a Menéndez Pelayo.

N. ${ }^{\circ}$ 36. Plaza de Legazpi.

N. ${ }^{\circ} 37$. Puerto de Toledo.

N. ${ }^{\circ}$ 38. Avda. Bruselas, esquina a Bonn. 
N. ${ }^{\circ}$ 39. Plaza Fernández Ladreda.

N. ${ }^{\circ}$ 40. Moratalaz. Plaza Pablo Garnica.

N. ${ }^{\circ}$ 41. San Diego, esquina a Martín Alvarez.

N. ${ }^{\circ}$ 42. Plaza de Roma.

N. ${ }^{\circ}$ 43. Goya, esquina a Alcalá.

N. ${ }^{\circ}$ 44. Juan Bravo, esquina a Serrano.

N. ${ }^{\circ}$ 45. Plaza Cánovas del Castillo.

N. ${ }^{\circ}$ 46. Avda. de José Antonio, esquina a Salud.

N. ${ }^{\circ}$ 47. Sol.

N. ${ }^{\circ}$ 48. Preciados. 


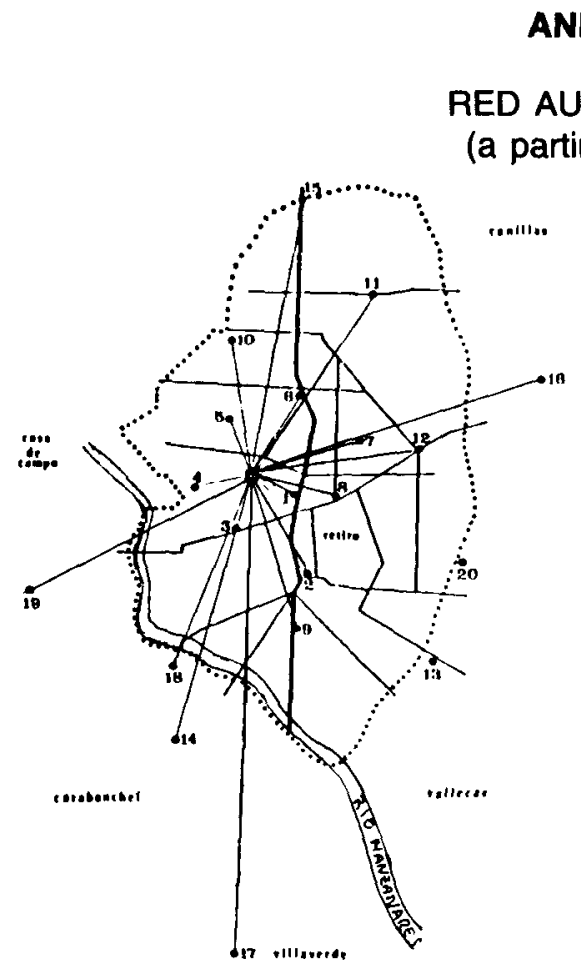

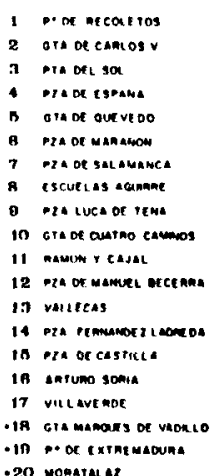

- go monatalaz

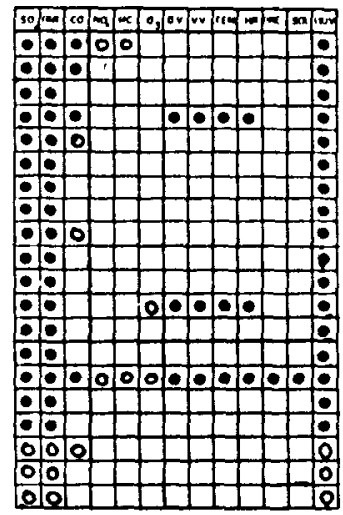

- Sensores a instalar en próxima ampliación

- ESTACION REMOTA

.. ZONA DE PAOTECCION ESPECIAL. Decreto 633/75. Perimetro delimitado por las siguientes vias. Plaza de Castilla - Calle de Bravo Murillo - Avenida de la Rerne Victoria - Calle dei General loañez de tbero. Paseo de San Francisco de Sales - Calles de isaac Peral y Fornandez de los Rios - Plaza de los Martires de Madnd - Paseos de Morei y Pintor Rosales - Calie de

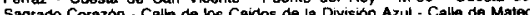
inurtia . Plara de Castilla.

\section{VISTA GENIERAL IDE UNA ESJACIONN REMOTA}

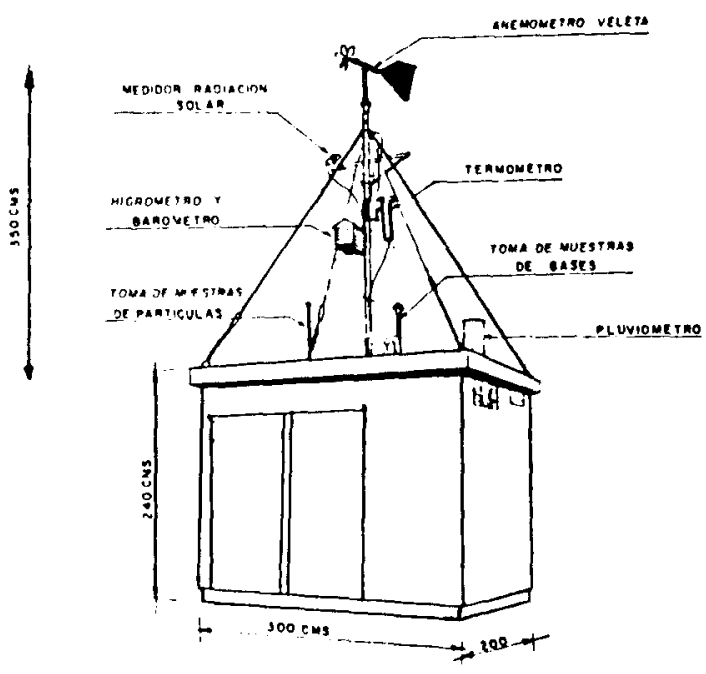


ANEXO 4. VALORACIÓN DE LOS NIVELES DE $\mathrm{SO}_{2}$ SOBRE LA SALUD

Concentración en la atmósfera de $\mathrm{SO}_{2}$

Según Lehmann y Hess:

Dosis mortal en $1 / 2$ - 1 hora

$1.400-1.700$
$400-500$
$170-240$
$60-100$
$20-30$

Dosis peligrosa en $1 / 2-1$ hora

ora, $\sin$ consecuencias

inmediatas o diferidas

$20-30$

Dosis soportable durante 6 horas con molestias pero

sin consecuencias graves

Según Zeehuissen

Dosis soportable durante sólo 3 minutos

300

Soportable durante sólo 1 minuto

Según Henderson y Haggart

Acusable por el olor

$8-13$

Irritante instantaneamente para los ojos

50

Máxima admisible para locales de trabajo

26

Máxima soportable en 1/2 - 1 hora

$130-260$

Peligroso en poco tiempo

$1.000-1.300$

Los valores que considera la OMS como causantes de efectos adversos son para medias diarias: más de $500 \mu \mathrm{g} / \mathrm{m}^{3}$ : excedentes de mortalidad y hospitalizaciones; de $200-500 \mu \mathrm{g} / \mathrm{m}^{3}$, agravamiento de enfermedades pulmonares; de $100 \mu \mathrm{g} / \mathrm{m}^{3}$, síntomas respiratorios; y de $80 \mu \mathrm{g} /$ $\mathrm{m}^{3}$, efectos sobre la visibilidad.

Según la EPA (Environmetal Protección Agency) el límite máximo es de $0,03 \mathrm{ppm}$ de promedio anual.

La OMS, en 1963, fijó cuatro niveles de índice de pureza del aire, que son los siguientes:

Nivel I: La concentración y la duración de la exposición son iguales o inferiores a los valores para los que, en el actual estado de nuestros conocimientos, ningún efecto directo o indirecto (incluida una modificación de reflejos o reacciones de adaptación o protección) puede ser observado. 
Nivel II: Las concentraciones y duraciones de exposición son iguales o superiores a los valores para los que se observará probablemente una irritación de los órganos de los sentidos, efectos nocivos para la vegetación, reducción de visibilidad u otros efectos desfavorables según el medio.

Nivel 1II: Las concentraciones y las duraciones de exposición son iguales o superiores a los valores para los que habrá probablemente, o bien un ataque a las condiciones fisiológicas vitales o bien alteraciones que pueden desencadenar enfermedades crónicas e incluso una muerte prematura.

Nivel IV: Las concentraciones y las duraciones de exposición son iguales o superiores a los valores para los que, probablemente, habrá enfermedad aguda o muerte prematura en los grupos vulnerables de la población. 